\title{
Influence of Surface Morphology on the Optical Properties of Antireflection Coating Formed by Porous Silicon Layer and ZnO Nanocoulms/Porous Silicon
}

\author{
S.M. Thahab \\ Nanotechnology and Advanced Materials Research Unit (NAMRU), Faculty of Engineering, \\ University of Kufa, 00964-21 Najaf, Iraq
}

\begin{abstract}
In this research the enhance the performance of solar cell efficiency by incorporating a system of Porous Silicon (PS) layer and $\mathrm{ZnO}$ Nanorode (NR) on the main surface textures of bulk silicon by electrochemical etching is carried out and investigated. The PS layers were prepared by electrochemical anodization. The optical properties of bulk $\mathrm{Si}, \mathrm{PS}$ and $\mathrm{ZnO}$ NR/PS layer was compared. Reflectivity spectrum exhibited assess the effectiveness of the PS and $\mathrm{ZnO}(\mathrm{NR})$ layers as antireflection covering bulk Si surface. The refractive index in the spectral range from $200-850 \mathrm{~nm}$ have been investiaged. It is observed that the PS layers could adjust the refractive indices for this structure. Furthermore, reflecting light through those layers gives a powerful refractive index by considering of a gradient-porosity of PS layer.
\end{abstract}

Key words: Reflectivity, $\mathrm{ZnO}$, nanostructures, porous silicon, $\mathrm{Si}$, refractive

\section{INTRODUCTION}

The utilization of Porous Silicon(PS) likewise Anti Reflection Covering (ARC) in view those refractive indices determinedly rely on the porosity (a bringing down of the reflectance in the affect ability extent from applying for solar cell which expanded those impede current thickness. The band gap shifts starting with $1.47 \mathrm{eV}$ to gravimetric thickness over $40-45 \%$ up to $1.8 \mathrm{eV}$ likewise the thickness for pores will be expanded (Dubey, 2013; Shokrollahi et al., 2012). PS might make used to change over higher energy sunlight based on radiation under those more extended wavelength light which may be consumed additional effectively under greater part silicon. In addition, there may be a expand in the sun based cell area the innovation for PS might be translated to multi-crystalline silicon which may be the material should make utilized for future PV provision. In the type electrochemical process, we could at the same time obtain antireflection coating, surface passivation. Also, substantial about deceased layer diffused locale (Alwan and Ali, 2015).

On the other hand, there are a few issues should consider for the utilization from applying for PS to sun based phones Helter Skelter resistivity of the PS. Furthermore, the effectiveness of electric transport the PS may be mechanically tantalizing on more need a poor kind conductivity (Lee et al., 2008). To get fancied optical properties of PS, it will be vital will think its refractive index. In addition, in this research is to consider a gradient-porosity PS layer which have been created and analyzed. The approaching about structure need a providing for fabricating graded-index optical waveguides. An efficient Anti Reflection Coating (ARC) can enhance solar cell performance through increased light coupling. Here, we investigate thermal evaporation in an atmosphere $\mathrm{ZnO}$ nanostructures as ARCs for $\mathrm{Si}$ solar cells and compare them to conventional single layer ARCs. ZnO NCs arrays display a broadband reflection suppression from 400-1200 nm (Weiying et al., 2011; Eswar et al., 2014; Pap et al., 2006).

\section{MATERIALS AND METHODS}

Experiment: N-type Si (111) wafer is ultrasonically cleaned with acetone and Isopropyl Alcohol (IPA) and then rinsed with distilled then cut to $2 \times 2 \mathrm{~cm}^{2}$ pieces and placed on a metal disk and sealed through an O-ring, so that, only the front side of the sample is exposed to the electrolyte as it described in our published paper (Thahab, 2011). This cell which is shown in Fig. 1 is the most widely used because it leads to uniform PS layers, allows an easy control of both porosity and thickness and it is suitable for front side illumination of the sample during the eaching. $\mathrm{ZnO} \mathrm{NCs}$ was prepared as it described in Thahab (2011).

The reflectance measurements were carried with PG10 spectrophotometer T70 (MR181-1 Specular Reflection) incidence angle: $5^{\circ}$ and in the spectral range $(200-850 \mathrm{~nm})$ with an ordinary rate reflectance frill and aligned UV-improved mirrors for use as extensions. Specular reflectance accessory is designed to measure the mirror-like reflectance from a sample surface. Specular reflectance is useful in the characterization of surfaces

Corresponding Author: S.M. Thahab, Department of Electronics and Communication, Faculty of Engineering, 


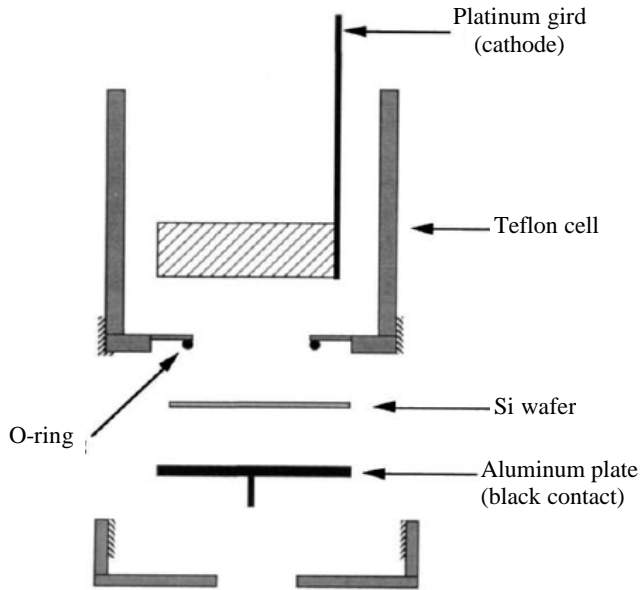

Fig. 1: Cross-sectional view of a conventional single-tank cell

such as mirrors or gloss paints. In addition, it can be used to determine the degree of polishing of an optical surface or to measure the film thickness of thin coatings. The experimental study of the refractive index of the PS layers has been performed by reflectance measurements as a function of wavelength in the range of $200-850 \mathrm{~nm}$ for the different samples.

\section{RESULTS AND DISCUSSION}

SEM image of the Porous Silicon (PS) substrate is revealed in Fig. 2. It can be seen that the surface of the PS essentials a sponge-like structure which comprises about various conceal dependence joined nano-pore of nano-silicon crystals for the homogeneous extensive range of $50 \mathrm{~nm}$. Those spreading of the pores was random in their distribution. It similarly might make viewing also of a nanocrystalline (quantum sponge) of an a system of pores (Feng et al., 2010). Because of this special structure, another property of the porous silicon is that the PS showed a very large inner surface area (of the order of $500-1000 \mathrm{~m}^{2} / \mathrm{cm}^{3}$ ). Thus, a large inner surface a large adsorption was expected for PS surface (Ahsanulhaq et al., 2007; Yubuta et al., 2007; Feng et al., 2010; Wang, 2004).

Figure 3 a shows an AFM image of bulk Si which has a flat surface morphology. The AFM result of the PS surface is shown in Fig. 3b. It can be seen that the random distribution over the entire surface of PS which would act as nucleation sites which inducing $\mathrm{ZnO} \mathrm{NCs}$ to grow along the preferred orientation. The surface morphology of the $\mathrm{ZnO} \mathrm{Ncs} / \mathrm{PS}$ film measured by AFM which is shown in Fig. 3c. From the AFM surface morphology

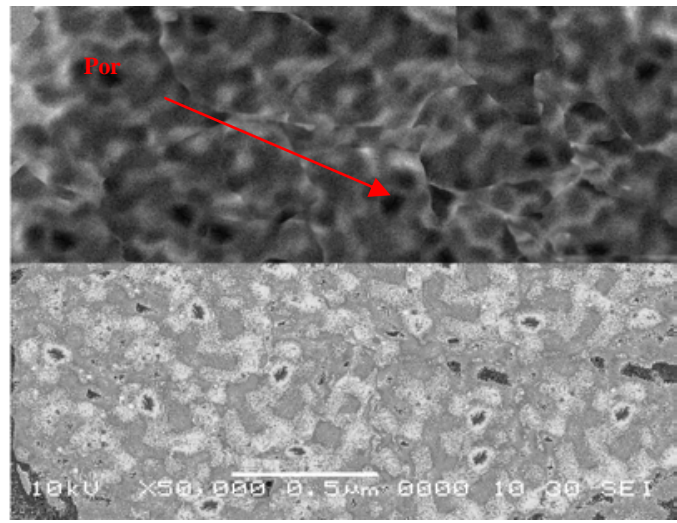

Fig. 2: SEM of PS/Si substrate

measurement of the PS and $\mathrm{ZnO} \mathrm{NCs} / \mathrm{PS}$ surface, the RMS roughness are found to increase from $2.55 \mathrm{~nm}$ for the PS to $6.08 \mathrm{~nm}$ for the $\mathrm{ZnO} \mathrm{NCs}$. The increase in the RMS roughness is attributed to the partial filling and formations of $\mathrm{ZnO} N \mathrm{~N}$ in the pores of PS. The surface of the porous silicon layer is composed of numerous nano-silicon crystals (Shishido et al., 2007).

The refractive index $(n)$, the extinction coefficient $(k)$ of PS substrate was calculated as below: The extinction coefficient (k) of PS was calculated using Eq. 1:

$$
K=\frac{\alpha \lambda}{4 p}
$$

where, $\lambda$ is the wavelength. The refractive index of PS was calculated using Eq. 2:

$$
\mathrm{n}=\left[\left(\frac{1+\mathrm{R}}{1+\mathrm{R}}\right)^{2}\left(1+\mathrm{K}^{2}\right)\right]^{1 / 2}+\left(\frac{1+\mathrm{R}}{1-\mathrm{R}}\right)
$$

Where:

$\mathrm{k}=$ The extinction coefficient

$\mathrm{R}=$ The optical reflectance

Figure 4 shows optical Reflectance (R), Absorbance (A), the real dielectric constant and the extinction coefficient (k) of PS nanostructure. It was noted that the real dielectric constant decreased and the extinction coefficient $\mathrm{k}$ increased with the decreasing in photon energy. A rapid increased were followed at lower photon energy followed by variable peaks at corresponding energies of the energy band gap, followed by a reduction at higher photon energy. Nevertheless, the reflectivity of PS nanostructures increased at higher wavelength value and decreased at short wavelengths due to the surface morphology of the PS nanostructures. 

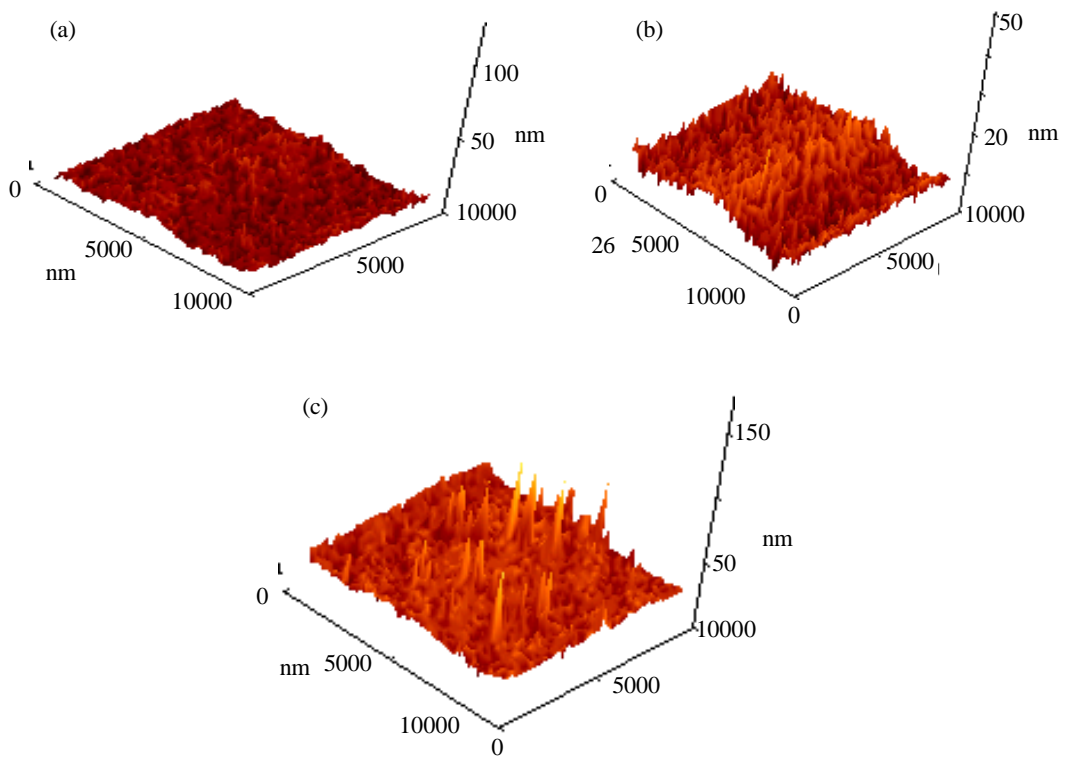

Fig. 3: AFM images of; a) Bulk pure Si; b) PS and c) ZnO NCs/PS

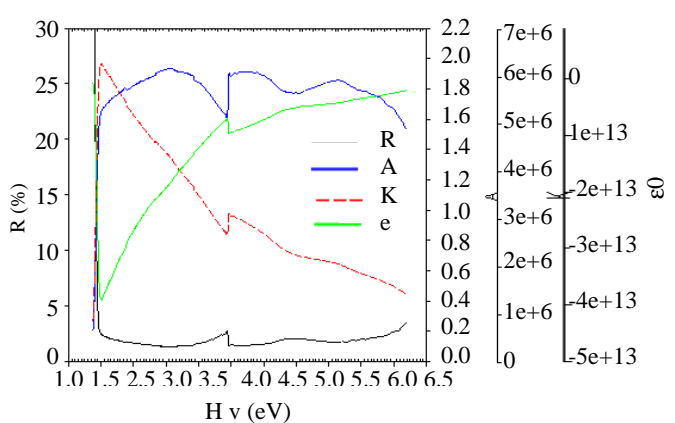

Fig. 4: Optical Reflectance (R), Absorbance (A), the real dielectric constant and the extinction coefficient $(\mathrm{k})$ PS nanostructure

Figure $5 \mathrm{a}, \mathrm{b}$ shows the reflect : and absorbance characteristics of the polished Si su : : PS textured and $\mathrm{ZnO} \mathrm{NCs/PS}$, respectively. It can $\mathrm{t}$ en that PS layer effectively decreases of reflectiv : below $800 \mathrm{~nm}$. The minimum reflectivity of PS 1 , is in the range (800-500) nm In compare with antire: : on ( $\mathrm{ZnO}$ ) NC, PS layer produced an evidently decrease of reflectivity for short wavelengths below $800 \mathrm{~nm}$. That reveals that $\mathrm{ZnO}$ $\mathrm{NCs}$ could be used as antireflection coating in solar cells, make its preparation is much simpler to implement and less expensive than multilayer antireflection coating like ( $\mathrm{ZnO}$ or $\mathrm{TiO}_{2}$ ) which require an expansive deposition system. The formation of PS layer could be improved controlling of the textured surface the PS layer in which it leads to decreases of reflectivity from the main partition of sensitivity (400-1000 nm) of silicon solar cell (Wang et al., 2004; Umar and Hahn 2006; Tang et al., 2009).
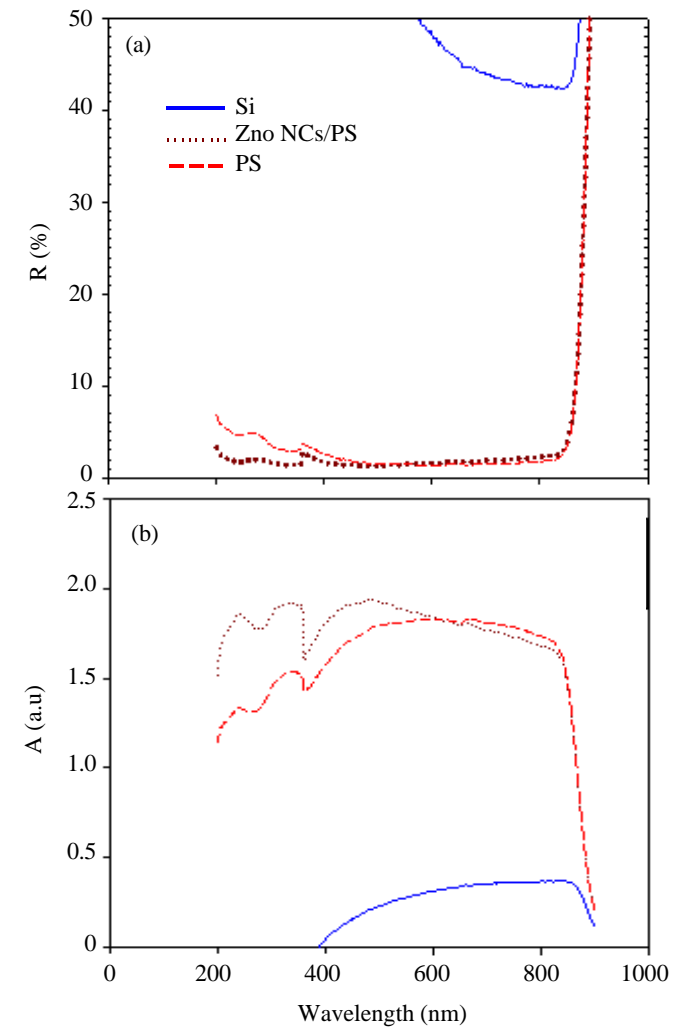

Fig. 5: a) Reflectivity and b) Absorbance spectra of bulk $\mathrm{Si}, \mathrm{PS}$ and $\mathrm{ZnO} \mathrm{NCs} / \mathrm{PS}$ as a function of wavelength

The two the majority essential trademark of a porous silicon layer need utility its refractive index and thickness. 


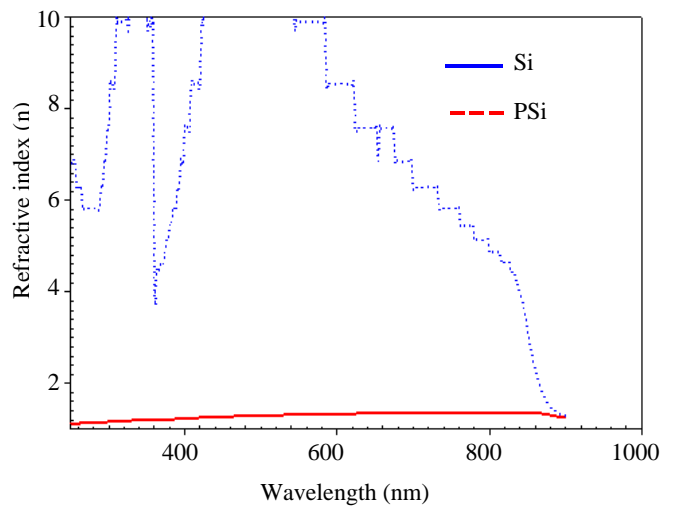

Fig. 6: Refractive index of bulk $\mathrm{Si}$ and PS as a function of wavelength

The weakening of the reflectivity may be because of diffusing also the transmission through the porous layer and the interfaces. This parameter need its important part in place to upgrade those light transformation effected of solar cell performance.

The refractive index turn of porosity of the Si layer resulting in the unconventionality measurement of reflectivity. Figure 6 shows the variation of refractive index as a function of wavelength. It is clear to observe that the $\mathrm{n}$ value in the range (850-200) $\mathrm{nm}$ of the PS layer exhibits two typical regions. In longer wavelengths, the refractive index exhibit an increasing and a wide amount of fringes because of the drop in the absorption in the layer might have been due to starting with the main surface about PS layer and PS/Si interface. The tapering leads to impedance matching between $\mathrm{Si}$ and air through a gradual reduction of the effective refractive index away from the PS surface.

\section{CONCLUSION}

The reflectivity spectra $\mathrm{PS}$ and $\mathrm{ZnO}(\mathrm{NCs}) / \mathrm{PS}$ layers were described. The low reflection PS and $\mathrm{ZnO} \mathrm{NCs} / \mathrm{PS}$ layers will be helpful as antireflection covering for solar cell application. The refractive index of the porous silicon layer builds which declines individually. PS layers demonstrated nanostructures with an ordinary characteristic size about regarding $40 \mathrm{~nm}$ for those pore diameters and vertically adjusted Si pillars. The reflection ranges utilized a crucial optical range in the $200-850 \mathrm{~nm}$. Optical tapering leads to a gradual reduction of the effective refractive index away from the surface, resulting in a low reflection, particularly at longer wavelengths and eliminating interference fringes through roughening of the air $\mathrm{ZnO}(\mathrm{NCs})$ interface.

\section{ACKNOWLEDGEMENTS}

The researchers would like to acknowledge the assistance offered. Nanotechnology and Advanced Materials Research Unit (NAMRU), Faculty of Engineering, University of Kufa, Kufa/Iraq

\section{REFERENCES}

Ahsanulhaq, Q., A. Umar and Y.B. Hahn, 2007. Growth of aligned $\mathrm{ZnO}$ nanorods and nanopencils on $\mathrm{ZnO} / \mathrm{Si}$ in aqueous solution: Growth mechanism and structural and optical properties. Nanotechnol., 18: 1-8.

Alwan, A.M. and S.M. Ali, 2015. Gradient-Porosity Porous Silicon (GPSi) as anti-reflection coating in solar cells applications. Eng. Technol. J., 33: 152-159.

Dubey, R.S., 2013. Electrochemical fabrication of porous silicon structures for solar cells. Nanosci. Nanoeng., 1: $36-40$.

Eswar, K.A., F.S. Husairi, A.A. Aziz, M. Rusop and S. Abdullah, 2014. Growth of $\mathrm{ZnO}$ nanosturctures on porous silicon in different concentration of $\mathrm{Zn} 2+$ Ion. Adv. Mater. Res., 832: 691-694.

Feng, L., A. Liu, M. Liu, Y. Ma and J. Wei et al., 2010. Synthesis, characterization and optical properties of flower-like $\mathrm{ZnO}$ nanorods by non-catalytic thermal evaporation. J. Alloys Compd., 492: 427-432.

Lee, Y.J., D.S. Ruby, D.W. Peters, B.B. McKenzie and J.W. Hsu, 2008. ZnO nanostructures as efficient antireflection layers in solar cells. Nano Lett., 8: 1501-1505.

Pap, A.E., K. Kordas, J. Vahakangas, A. Uusimaki and S. Leppavuori et al., 2006. Optical properties of porous silicon Part III: Comparison of experimental and theoretical results. Opt. Mater., 28: 506-513.

Shishido, T., K. Yubuta, T. Sato, A. Nomura and J. Ye et al., 2007. Synthesis of Zinc oxide fibers from precursor Bis(acetylacetonato)Zinc: Tracking the mineralization process and micro- and nano-structural changes. J. Alloys Compd., 439: 227-231.

Shokrollahi, A., M. Zare, A. Mortezaali and S.S. Ramezani, 2012. Analysis of optical properties of porous silicon nanostructure single and gradient-porosity layers for optical applications. J. Appl. Phys., 112: 053506-1-053506-6.

Tang, H., J.C. Chang, Y. Shan, D.D.D. Ma and T.Y. Lui et al., 2009. Growth mechanism of $\mathrm{ZnO}$ nanowires via direct $\mathrm{Zn}$ evaporation. J. Mater. Sci., 44: $563-571$.

Thahab, S.M., 2011. Preparation and structural characterizations of $\mathrm{ZnO}$ nano-columns grown on porous silicon-silicon (PS/Si (111)) by thermal evaporation. Optoelectron Adv. Mater. Rapid Commun., 5: 1107-1110. 
Umar, A. and Y.B. Hahn, 2006. ZnO nanosheet networks and hexagonal nanodiscs grown on silicon substrate: Growth mechanism and structural and optical properties. Nanotechnol., 17: 2174-2180.

Wang, Z., X.F. Qian, J. Yin and Z.K. Zhu, 2004. Large-scale fabrication of tower-like, flower-like and tube-like $\mathrm{ZnO}$ arrays by a simple chemical solution route. Langmuir, 20: 3441-3448.
Wang, Z.L., 2004. Zinc oxide nanostructures: Growth, properties and applications. J. Phys.: Condens. Matter, 16: R829-R858.

Weiying, O., Z. Lei, D. Hongwei, Z. Jun and W. Wenjing, 2011. Optical and electrical properties of porous silicon layer formed on the textured surface by electrochemical etching. J. Semicond., 32: 1-4.

Yubuta, K., T. Sato, A. Nomura, K. Haga and T. Shishido, 2007. Structural characterization of $\mathrm{ZnO}$ nano-chains studied by electron microscopy. J. Alloys Compd., 436: 396-399. 